\title{
Frontières
}

\section{La logique cannibale de Montaigne}

\section{Irène Salas et Flore Marie}

Volume 23, numéro 1, automne 2010

Enquêtes sur le cadavre : 1. Fascination

URI : https://id.erudit.org/iderudit/1004022ar

DOI : https://doi.org/10.7202/1004022ar

Aller au sommaire du numéro

\section{Éditeur(s)}

Université du Québec à Montréal

ISSN

1916-0976 (numérique)

Découvrir la revue

Citer cet article

Salas, I. \& Marie, F. (2010). La logique cannibale de Montaigne. Frontières, 23(1), 47-53. https://doi.org/10.7202/1004022ar

\section{Résumé de l'article}

La logique cannibale - telle que l'interprète Montaigne - consiste à perdre son corps pour mieux cristalliser la parole et faire durer la mémoire. Bien plus qu'un simple mode de communication, pour les Tupinambas, le corps représente une véritable économie (un commerce) : il se transforme en un signe qui permet l'échange symbolique. Grâce à cette consommation absolue, la mort devient régénératrice. Les Essais de Montaigne sont eux-mêmes un corps vivant puisqu'ils servent de lieu de rencontre et synthétisent les échanges avec d'autres corps. Montaigne se « nourrit » littéralement de l'Autre afin d'affermir son propre corps et de l'empêcher de se dégrader. Comme le cannibale, les humanistes de la Renaissance pratiquent l' "innutrition » : ils consomment le corps mort de leurs aînés (le corps textuel des Anciens) pour exister dans la postérité. 


\section{Résumé}

La logique cannibale - telle que l'interprète Montaigne - consiste à perdre son corps pour mieux cristalliser la parole et faire durer la mémoire. Bien plus qu'un simple mode de communication, pour les Tupinambas, le corps représente une véritable économie (un commerce): il se transforme en un signe qui permet l'échange symbolique. Grâce à cette consommation absolue, la mort devient régénératrice. Les Essais de Montaigne sont eux-mêmes un corps vivant puisqu'ils servent de lieu de rencontre et synthétisent les échanges avec d'autres corps. Montaigne se "nourrit» littéralement de I'Autre afin d'affermir son propre corps et de l'empêcher de se dégrader. Comme le cannibale, les humanistes de la Renaissance pratiquent I' "innutrition»: ils consomment le corps mort de leurs aînés (le corps textuel des Anciens) pour exister dans la postérité.

Mots clés: Montaigne - cannibalisme cadavre - corps - innutrition - mémoire.

\section{Abstract}

Montaigne's cannibal logic consists of losing the body to crystallize better the word and prolong the memory. For the Tupinambas, the body, much more than a simple communication medium, represents a real economy (a trade good): it is transformed into a sign which enables a symbolic exchange. Thanks to this absolute consumption, death becomes regenerative. Montaigne's Essays are themselves a living body; they serve as a meeting place and synthesize exchanges with other bodies. Montaigne literally "feeds" on the Other to strengthen his own body and to protect it from degradation. Like cannibals, the humanists of the Renaissance practice "innutrition": they consume the dead bodies of their elders (the textual bodies of the Ancients) in order to preserve themselves for posterity.

Keywords: Montaigne - cannibalism corpse - body - innutrition - memory.

\section{LA LOGIQUE CANNIBALE DE MONTAIGNE}

Irène Salas,

doctorante, EHESS Paris (Arts et Langages),

Flore Marie,

professeure certifiée, Sorbonne-Nouvelle Paris III (Littérature française et Linguistique).

Qu'en est-il du traitement du cadavre à la Renaissance? Cette grande époque d'investigation des mystères du corps humain voit se développer la pratique de la dissection humaine portant les anatomistes au cœur de la corporis fabrica; d'autre part, elle voit se multiplier à partir du XVI siècle les cabinets de curiosités où sont exposés des fragments de dépouilles, des préparations veineuses ou nerveuses, des crânes, des squelettes, des fœtus, des tumeurs... Qu'en est-il du statut du cadavre dans les représentations artistiques et littéraires? Peut-on parler en particulier d'une «poétique» du cadavre chez Michel de Montaigne?

Ainsi, dans un chapitre des Essais (1580, I, XXXI), Montaigne imagine une logique cannibale qui consiste à perdre son corps pour mieux cristalliser la parole et faire durer la mémoire. Bien plus qu'un simple mode de communication, le corps représente une véritable économie (un commerce) dans la culture cannibale. Ainsi le don, comme il se pratique dans les sociétés primitives, passe toujours par le corps et doit même idéalement déboucher sur un «don du corps ». Il est possible de considérer l'anthropologie du cannibale comme un «don forcé» de soi, une offrande ou un sacrifice du corps individuel nécessaire au maintien du corps social. Le corps cannibale se transforme dès lors en un signe qui permet l'échange symbolique. Grâce à cette consommation absolue, la mort devient régénératrice.

De même, en offrant son corps - ou plus exactement son texte - en nourriture au lecteur, l'auteur investit la mémoire des vivants.

\section{LE CADAVRE COMME RÉGÉNÉRATION}

«Des Cannibales»: ce célèbre chapitre des Essais de Montaigne, qui semble annoncer l'expression de la sauvagerie la plus inhumaine, fait au contraire l'apologie d'une société très harmonieuse: «Je trouve [...] qu'il n'y a rien de barbare et de sauvage en cette nation, à ce qu'on m'en 
LES VRAIS BARBARES SONT DONC LES EUROPÉENS QUI SE LIVRENT

AUX GUERRES CIVILES, À L'ORIGINE DE SANGLANTS MASSACRES. LE VRAI SUPPLICE POUR MONTAIGNE NE CONCERNE PAS CELUI QUI MANGE UN HOMME - UNE FOIS TRÉPASSÉ -, NI CELUI QUI EST CAPABLE DE TORTURE SUR SON PROCHAIN; LE PIRE EST DE «MANGER UN HOMME VIVANT» (MONTAIGNE, 1580, I, XXXI;). IL FAIT UNE BRÈVE ALLUSION À CEUX QUI, DU VIVANT DE LEUR VICTIME, LA TOURMENTENT COMME DES VAUTOURS S'ACHARNANT SUR UNE PROIE AFFAIBLIE MAIS TOUJOURS PALPITANTE. IL ÉVOQUE LES BANQUIERS ET LES USURIERS AVIDES, DONT IL DÉPLORE LES MÉTHODES ET LES EXACTIONS.

a rapporté: sinon que chacun appelle barbarie, ce qui n'est pas de son usage ${ }^{1}$. Le terme semble fondé sur l'onomatopée primitive «bar-bar-bar »; déjà les Grecs nommaient «barbare» tous ceux qui n'étaient pas Hellènes. Une question se pose alors : ces Indiens qualifiés de «sauvages » ou de «barbares", auxquels certains conquistadores déniaient une âme à cause de leur pratique du cannibalisme - légitimant ainsi leur évincement du champ de l'humanité -, peuvent-ils vraiment être compris par l'Européen du $\mathrm{XVI}^{\mathrm{e}}$ siècle? L'opacité de leur langage, de leurs mœurs, de leurs us et coutumes est-elle impénétrable ? Face à l'«estrange», Montaigne lui-même prend mille précautions rhétoriques quant à sa «capacité» à les appréhender: "J'ay peur que nous avons les yeux plus grands que le ventre, et plus de curiosité que nous n'avons de capacité. Nous embrassons tout, mais nous n'étreignons que du vent. » Cet appétit de comprendre l'Autre n'est-il pas pure chimère? La pensée européenne peut-elle conceptualiser le cannibalisme en dehors de toute logique de survie ${ }^{2}$ ?

Face à la monstruosité du préjugé, c'est tout en douceur que Montaigne essaie de passer la frontière du territoire «barbare» afin d'entrer dans l'inconnu de ce Nouveau Monde. Il est plus méfiant envers son propre regard qu'envers l'Autre. En témoignent les nombreux modalisateurs qui jalonnent sa prose («mais», «je ne sais si », «il semble», «sinon que», « et si pourtant») et qui révèlent son trouble face à l'inédit des récits de voyages qui lui sont rapportés. Pour comprendre l'Autre - que Montaigne n'a jamais rencontré de visu -, il est indispensable de se fondre en lui, de le contextualiser le plus précisément pos- sible et donc de se nourrir de force détails puisés dans des récits tant écrits qu'oraux. Aussi Montaigne décrit-il scrupuleusement le profil de ses témoins, dont il vante la simplicité et l'innocence des déclarations, qualités garantes de leur authenticité. Il s'agit en effet d'«homme[s] très fidèle[s], ou si simple[s] qu'il[s] n'ai[ent] pas dequoy bastir et donner de la vray-semblance, à des inventions fauces» et qui sont plus à même de fournir un "veritable tesmoignage». Il multiplie les points de vue et se renseigne auprès de «matelots» et de «marchands »; enfin, il se fie à ses sens : «il m'a fait veoir », «j'en ay tasté »... Ainsi, les «topographes» prennent pour Montaigne l'avantage sur les «cosmographes», qui le laissent sceptique parce qu'ils ne peuvent s'empêcher d'englober le monde dans sa plénitude et altèrent leur vision du singulier pour le plier aux exigences d'une harmonie cosmique: «car les fines gens remarquent bien plus curieusement et plus de choses, mais ils les glosent; et, pour faire valoir leur interpretation et la persuader, ils ne se peuvent garder d'alterer un peu l'Histoire» (Montaigne, 1580, I, XXXI).

L'homme simple est la passerelle permettant de combler le grand écart spatio-temporel entre deux réalités anachroniques: "J'ay eu long temps avec moy un homme qui avoit demeuré dix ou douze ans en cet autre monde qui a esté descouvert en nostre siecle [...] (Montaigne, 1580, I, XXXI; nous soulignons). La paronomase «autre» / «nostre» réduit cependant ce vertige et laisse entrevoir la possibilité, par glissement phonétique, de l'interpénétration de ces deux mondes apparemment inconciliables.
Certes, l'usage d'un corps défunt n'est pas un fait nouveau en Europe. Montaigne rapporte le récit d'un guerrier qui réclame que sa dépouille soit bouillie pour décoller la chair du squelette, afin que les futurs guerriers portent ses os comme reliques dans les combats à venir :

Édouard premier, Roy d'Angleterre,

[...] mourant, obligea son fils par solennel serment à ce qu'estant trespassé, il fist bouillir son corps pour desprendre sa chair d'avec les os, laquelle il fit enterrer; et quant aux os, qu'il les reservast pour les porter avec luy et en son armée, toutes les fois qu'il luy adviendroit d'avoir guerre contre les Escossois (Montaigne, 1580, I, III).

De son côté, Jean de Léry (1578) relate des rites similaires chez les indigènes qu'il a observés au Brésil: les ennemis sont intégralement mangés, excepté la cervelle; sont ensuite érigés des monticules de têtes à l'entrée de leur village, manière de dire la bravoure des guerriers. Les grands os servent à fabriquer des flûtes, quant aux dents, elles deviennent des colliers ostensiblement portés en signe de victoire. La notion de barbarie est ainsi étroitement liée au traitement du cadavre ; c'est pourtant bien dans cette optique que Montaigne relativise la prétendue barbarie des Tupinambas du Brésil. Il se demande s'il est pire de manger un mort ou d'infliger au vivant d'affreuses souffrances ${ }^{3}$ :

Je pense qu'il y a plus de barbarie à manger un homme vivant qu'à le manger mort, à deschirer, par tourmens et par geénes, un corps encore plein de sentiment, le faire rostir par le menu, le faire mordre et meurtrir aux chiens et aux pourceaux (comme nous l'avons, non seulement leu, mais veu de fresche memoire, non entre des ennemis anciens, mais entre des voisins et concitoyens, et, qui pis est, sous pretexte de pieté et de religion), que de le rostir et manger apres qu'il est trespassé (Montaigne, 1580, I, XXXI).

D'ailleurs, Montaigne note qu'au contact des conquistadores, les Indiens adopteront les tortures des Portugais, comprenant que la souffrance y est plus cruelle:

Ayant apperçeu que les Portuguois, qui s'estoient r'alliez à leurs adversaires, usoient d'une autre sorte de mort contre eux, quand ils les prenoient, qui estoit de les enterrer jusques à la ceinture, et tirer au demeurant du corps force coups de traict, et les pendre apres : ils penserent que ces gens icy de l'autre monde [...] ne prenoient pas sans occasion cette sorte 
de vengeance, et qu'elle devoit estre plus aigre que la leur, commencerent de quitter leur façon ancienne pour suivre cette-cy (I, XXXI).

Les vrais barbares sont donc les Européens qui se livrent aux guerres civiles, à l'origine de sanglants massacres. Le vrai supplice pour Montaigne ne concerne pas celui qui mange un homme - une fois trépassé -, ni celui qui est capable de torture sur son prochain; le pire est de «manger un homme vivant» (Montaigne,

\section{UNE SOCIÉTÉ QUI RÉDUIT}

\section{CERTAINS DE SES MEMBRES}

\section{À L'ÉTAT DE DEMI-CADAVRE}

NE LAISSE-T-ELLE PAS

\section{SOUPÇONNER UNE FORME}

\section{D'ANTHROPOPHAGIE MASQUÉE ?}

1580, I, XXXI; nous soulignons). Il fait une brève allusion à ceux qui, du vivant de leur victime, la tourmentent comme des vautours s'acharnant sur une proie affaiblie mais toujours palpitante. Il évoque les banquiers et les usuriers avides, dont il déplore les méthodes et les exactions. La question de la sensibilité est centrale chez Montaigne: la douleur n'a selon lui aucune valeur si elle n'est ressentie par la «molle impression des sens » (Montaigne, 1580, II, VI). Ainsi en est-il du corps endormi et du corps mort. Le premier laisse affleurer la douleur, mais celle-ci n'est pas «à nous »; quant au corps mort, il n'appartient même plus au sujet alors anesthésié:

Il y a plusieurs animaux, et des hommes mesmes, apres qu'ils sont trespassez, ausquels on voit resserrer et remuer des muscles. Chacun sçait par experience qu'il y a des parties qui se branslent, dressent et couchent souvent sans son congé. Or ces passions qui ne nous touchent que par l'escorse, ne se peuvent dire nostres (II, VI).

Montaigne prend donc en considération le corps martyrisé à vif pour juger de la barbarie. On pourrait ainsi distinguer quatre états du corps: le corps vivant en pleine santé; le corps souffrant et atrophié; le corps endormi; le cadavre.

Dans les récits de Montaigne, nous pouvons constater que les Tupinambas ne connaissent pas ce corps souffrant, intermédiaire, puisque l'auteur ne manque pas de souligner que leurs prisonniers sont particulièrement bien traités et souriants: «Ils les traictent en toute liberté, affin que la vie leur soit d'autant plus chere»; plus loin: "pendant ces deux ou trois mois qu'on les garde, ils portent une contenance gaye» (Montaigne, 1580, I, XXXI) ${ }^{4}$.

Il est instructif aussi d'observer que Montaigne considère leur société comme un corps sain et vigoureux, celui d'un premier âge: "Ces nations me semblent donq ainsi barbares, pour avoir receu fort peu de façon de l'esprit humain, et estre encore fort voisines de leur naifveté originelle» (I, XXXI). Il leur oppose le second état des corps, soit la barbarie à l'européenne vue par le prisme des Indiens se rendant à Rouen, qui découvrent avec stupeur le dépérissement physique des couches les plus pauvres de la population:

Ils avoyent aperçeu qu'il y avoit parmy nous des hommes pleins et gorgez de toutes sortes de commoditez, et que leurs moitiez estoient mendians à leurs portes, décharnez de faim et de pauvreté; et trouvoient estrange comme ces moitiez icy necessiteuses pouvoient souffrir une telle injustice, qu'ils ne prinsent les autres à la gorge, ou missent le feu à leurs maisons (I,

XXXI).

En effet, sur leurs terres où la nature se montre foisonnante et généreuse, cette réduction à la misère n'existe pas: « $\mathrm{Au}$ demeurant, ils vivent en une contrée de païs tres-plaisante et bien temperée; de façon qu'à ce que m'ont dit mes tesmoings, il est rare d'y voir un homme malade; et m'ont asseuré n'en y avoir veu aucun tremblant, chassieux, edenté, ou courbé de vieillesse»(I, XXXI). Une société qui réduit certains de ses membres à l'état de demi-cadavre ne laisse-t-elle pas soupçonner une forme d'anthropophagie masquée? Encore une fois, Montaigne se demande où se situe réellement la barbarie et il étend sa vision du corps malade à l'ensemble de la civilisation occidentale atrophiée par ses vices:

Il ne se trouva jamais aucune opinion si desreglée qui excusat la trahison, la desloyauté, la tyrannie, la cruauté, qui sont nos fautes ordinaires. Nous les pouvons donq bien appeller barbares, eu esgard aux regles de la raison, mais non pas eu esgard à nous, qui les surpassons en toute sorte de barbarie. Leur guerre est toute noble et genereuse, et a autant d'excuse et de beauté que cette maladie humaine en peut recevoir (I, XXXI).

Le grand corps malade de la société occidentale apparaît vieilli, gris et usé, tandis que rayonne de tous ses feux la prime jeunesse de ce Nouveau Monde qui garde la vitalité de la Nature:

Ils sont sauvages, de mesmes que nous appellons sauvages les fruicts que nature, de soy et de son progrez ordinaire, a produicts : là où, à la verité, ce sont ceux que nous avons alterez par nostre artifice et detournez de l'ordre commun, que nous devrions appeller plutost sauvages. En ceux là sont vives et vigoureuses les vrayes, et plus utiles et naturelles vertus et proprietez, lesquelles nous avons abastardies en ceux-cy, et les avons seulement accommodées au plaisir de nostre goust corrompu (I, XXXI).

Pour Montaigne, le véritable état cadavérique est celui du corps (physique ou social) étouffé, comme si la société européenne, nourrie d'un sens de la civilisation frelaté, s'était fourvoyée au détriment de la nature et du réel:

Car il me semble que ce que nous voyons par experience en ces nations là, surpasse, non seulement toutes les peintures dequoy la poesie a embelly l'age doré, et toutes ses inventions à feindre une heureuse condition d'hommes, mais encore la conception et le desir mesme de la philosophie. Ils n'ont peu imaginer une nayfveté si pure et simple, comme nous la voyons par experience; ny n'ont peu croire que nostre societé se peut maintenir avec si peu d'artifice et de soudeure humaine (I, XXXI).

Pour l'humaniste Montaigne, la nature de l'homme est universelle et perdure à travers les âges et les différentes cultures ou civilisations. L'image de l'homme sauvage qu'il décrit joue comme un contrepoint réflexif sur ce que les Européens devraient ou pourraient être: donner à voir l'Autre permet pour l'auteur de mieux penser «nostre societé » et donc d'envisager avec optimisme le salut de l'Europe par l'autocritique de son égarement dans l'avidité. La férocité du propos envers les contemporains n'est que le pendant d'un désir de les éduquer: Montaigne prône le dépouillement du sauvage comme philosophie. Aussi est-il inspiré par l'ascétisme stoïcien, où simplicité et frugalité font écho aux modes de vie des Tupinambas qui «ne font autre repas que celuy là » et «mangent sans autre artifice [leurs viandes et leurs poissons] que de les cuire» (I, XXXI). D'ailleurs, Montaigne rappelle que les stoïciens ne voient aucun mal à pratiquer le cannibalisme lorsqu'il est une nécessité vitale:

Chrysippus et Zenon, chefs de la secte Stoicque, ont bien pensé qu'il n'y avoit aucun mal de se servir de nostre charoigne à quoy que ce fut pour nostre besoin, et d'en tirer de la nourriture: comme nos ancestres, estans assiegez par Caesar en la ville de Alexia, se resolurent de soustenir la faim de ce 
siege par les corps des vieillars, des femmes et autres personnes inutiles au combat (I, XXXI).

Si Montaigne légitime cette tradition, ce qu'il désapprouve en revanche est l'hybris et l'appétit des conquistadores, inconnu des Indiens qui se satisfont de la générosité de la nature: "Ils jouyssent encore de cette uberté naturelle qui les fournit sans travail et sans peine de toutes choses necessaires, en telle abondance qu'ils n'ont que faire d'agrandir leurs limites. [...] Tout ce qui est au-delà, est superflu pour eux» (I, XXXI).

En somme, le vrai cannibale n'est-il pas celui qui détruit sans possibilité de régénérescence, comme le font les Européens dans le Nouveau Monde? Rabelais développe une opinion parallèle à propos de la soif démesurée de conquête:

La manière d'entretenir et retenir pays nouvellement conquis n'est [pas] les peuples pillant, forçant, angariant, ruinant, mal vexant et régissant avec verges de fer; bref, les peuples mangeant et dévorant, en la façon que Homère appelle le roi inique Demovore, c'est-à-dire mangeur de peuple (Rabelais, 1552, Tiers Livre, I). Le cannibalisme de la Renaissance ne s'illustre-t-il pas dans les exactions commises par les conquistadores qui ont anéanti des civilisations entières (Aztèques, Mayas, Incas...) en quelques décennies? Montaigne oppose deux cannibalismes: l'un fécond, qui participe d'une dynamique cyclique; l'autre stérile, qui n'est qu'anéantissement et donc qui mérite le châtiment divin: "Dieu a meritoirement permis que ces grands pillages se soient absorbez par la mer en les transportant, ou par les guerres intestines dequoy ils se sont entremangez entre eux » (Montaigne, 1580, III, VI).

\section{LE CADAVRE COMME MÉDIATEUR}

Le cadavre est transactionnel: Montaigne témoigne ainsi des différentes formes d'offrandes et de dépècements du cadavre, en légitimant ces pratiques dès lors que la relique a valeur totémique. Ainsi, le démembrement peut:

- soit être assimilé au trophée de bataille: "Chacun raporte pour son trophée la teste de l'ennemy qu'il a tué, et l'attache à l'entrée de son logis » (Montaigne, 1580, I, III) ;

- soit avoir un rôle apotropaïque: «Jean Vischa [...] voulut qu'on l'escorchast apres sa mort et de sa peau qu'on fist un tabourin à porter à la guerre contre ses ennemis ${ }^{5}$; «Et d'autres peuples en ce mesme monde, trainent à la guerre les corps des vaillans hommes qui sont morts en leurs batailles, pour leur servir de bonne fortune et d'encouragement» (Montaigne, 1580, I, XXXI) ;

- soit servir de nourriture de célébration de victoire lors d'une assemblée : "Cela faict, ils le rostissent et en mangent en commun et en envoient des lopins à ceux de leurs amis qui sont absens» (I, XXXI);

- soit guérir: "Et les medecins ne craignent pas de s'en servir à toute sorte d'usage pour nostre santé » (I, XXXI).

Le cadavre à la Renaissance se pense à travers le paradigme du don, cher aux humanistes. Non seulement il est porteur d'un savoir, mais sa présence incite à la tempérance des vanités humaines. Point d'ancrage, il est sujet d'une exploration réflexive de l'homme, le régulant et le resituant à sa juste échelle, à sa mesure naturelle.

Dans la pratique de l'exocannibalisme des Tupinambas décrite par Montaigne, le cadavre apparaît comme une monnaie d'échange. En effet, lors d'assauts entre tribus voisines, les vainqueurs tiennent prisonniers leurs rivaux et n'attendent d'eux que l'acceptation verbale de leur défaite: ils veulent «arracher de leur bouche quelque parole molle ou rabaissée » (Montaigne, 1580, I, XXXI). Si les prisonniers refusent de délivrer cette parole, ils sont mis à mort puis mangés par leurs ennemis qui les " entretiennent communément des menasses de leur mort future, des tourmens qu'ils y auront à souffrir, des apprests qu'on dresse pour cet effect, du detranchement de leurs membres, et du festin qui se fera à leurs despens » (I, XXXI). La puissance du Verbe est ici décisive et représente un enjeu vital: car l'appropriation du corps de l'ennemi compense et remplace le refus de la parole. Cependant, le corps sacrifié est un moindre gain que l'aveu oral de la défaite et ne permet de sceller aucune victoire ni suprématie d'un groupe sur l'autre:

Ils ne demandent à leurs prisonniers

autre rançon que la confession et recognoissance d'estre vaincus; mais il ne s'en trouve pas un, en tout un siecle, qui n'ayme mieux la mort que de relascher, ny par contenance, ny de parole, un seul point d'une grandeur de courage invincible: il ne s'en void aucun qui n'ayme mieux estre tué et mangé, que de requerir seulement de ne l'estre pas (I, XXXI).

L'équilibre des forces inter-claniques est maintenu. De ce fait, le corps tribal de l'Autre n'est jamais absorbé: "Qui pour quelque dangier de la mort voisine ne relasche aucun point de son asseurance; qui regarde encores, en rendant l'ame, son ennemy d'une veue ferme et desdaigneuse, il est battu, non pas de nous, mais de la fortune; il est tué, non pas vaincu» (I,
XXXI). Le corps organisé du clan prime donc le corps de l'individu et le prisonnier n'hésite pas à s'auto-sacrifier pour assurer la pérennité de sa communauté: «ils pressent leurs maistres de se haster de les mettre en cette espreuve; ils les deffient, les injurient, leur reprochent leur lacheté et le nombre des batailles perdues contre les leurs », relate Montaigne (I, XXXI).

Les Tupinambas, comme leurs voisins, sont éduqués dans les mêmes règles éthiques et partagent un langage guerrier où le cannibalisme est intégré par les deux parties. Il ne s'agit aucunement d'un cannibalisme sauvage (tel qu'il peut apparaître aux Européens), mais d'un cannibalisme rationnel qui n'a donc rien d'effrayant. Hypercodifié, il est accepté par les victimes qui s'abandonnent aux aléas de leur fortune sans songer à se rebeller. Ce cannibalisme peut alors s'apparenter à un véritable acte contractuel qui scelle l'entente entre les deux adversaires. Il signifie: je te mange comme tu m'as mangé. Cette convenance tacite garantit la non-altération des parties et permet de préserver des relations saines et équilibrées entre les tribus : cette pratique subtile consiste à perdre une partie (le membre d'une tribu) pour ne pas perdre un tout (le corps communautaire). Cette forme d'anthropophagie est le garant de l'intégrité de la collectivité, puisque manger un ennemi compense les pertes de guerre. La chair n'est pas traitée sur le mode barbare, déchiquetée et dévorée, mais elle est un gage de pacification. Le cannibalisme de ces Indiens a donc une forte résonance symbolique. Il entretient une communication quasi filiale entre les tribus, puisque l'Autre est inscrit de manière ancestrale dans les entrailles du clan opposé, fait prisonnier au cours d'un précédent affront puis dévoré. La manducation de l'ennemi suggère donc un pacte charnel entre les deux groupes, un lignage, voire une communion qui n'est pas sans rappeler l'Eucharistie, où le corps du cannibale rejoint le corps du Christ - ce corps indestructible mangé indéfiniment et dont se nourrit et s'accroît, au fil des semaines, des années et des siècles, la communauté des fidèles :

[...] qu'ils viennent hardiment trétous et s'assemblent pour disner de luy: car ils mangeront quant et quant leurs peres et leurs ayeux, qui ont servy d'aliment et de nourriture à son corps. Ces muscles, dit-il, cette cher et ces veines, ce sont les vostres, pauvres fols que vous estes; vous ne recognoissez pas que la substance des membres de vos ancestres s'y tient encore: savourez les bien, vous y trouverez le goust de vostre propre chair (Montaigne, 1580, I, XXXI). 
LE CADAVRE EST AMBIGU: D'UNE PART, IL EST L'IMAGE INOUBLIABLE QUI DOIT AIDER À APAISER LE FEU DES VAINES PRÉTENTIONS HUMAINES; D'AUTRE PART, LA CONSCIENCE DE SON IMMINENCE NE DOIT PAS ENTRAVER LA VITALITÉ NI LA BRAVOURE, CAR L'ANGOISSE INSTINCTIVE ET PÉTRIFIANTE DE LA MORT EST LE POISON DES VIVANTS.

Ainsi contextualisé, le cannibalisme est le siège d'une transmigration énergétique, figurant un processus harmonieux. La construction de l'individu, pour être complète, doit intégrer l'Autre: en mangeant B les cannibales A assimilent non seulement l'étranger (l'ennemi, l'altérité), mais aussi leurs propres ascendants (fondements de leur identité) qui ont été mangés par B.

Si le cadavre est une image, il est aussi une fiction et la manducation de la chair humaine est une métaphorisation des relations entre les hommes. Il est un opérateur symbolique avec lequel la culture joue pour s'auto-instituer. Le cannibalisme est une institution - universelle sous sa forme réelle ou imaginaire - qui a un grand pouvoir d'inventer des significations, d'où son omniprésence dans l'univers merveilleux des contes (ogres, géants, loups-garous, etc.). Il modélise le social et a valeur anthropopoietique (permettant de faire, de construire, de fabriquer de l'humain).

Dans le contexte anthropologique que rapporte Montaigne, le cadavre a pour rôle de médiatiser les échanges interethniques et d'équilibrer la superstructure sociale; mais il est aussi immortalisé dans et par le vivant, ce qui brouille d'autant les frontières élastiques entre ces deux mondes.

\section{LE CADAVRE ANIMÉ: \\ SA PÉRENNITÉ DANS LE VIVANT}

Pour Montaigne, la vitalité s'exprime dans le courage et la fermeté, tandis que lâcheté et résignation sont nécrose. Autrement dit, le vrai cadavre ne gît pas toujours là où on croit: celui qui courbe l'échine et qui manque d'appétence pour la vie est un vivant-mort. À l'inverse, la mort héroïque fait éternellement briller autour du défunt l'aura lumineuse de sa vaillance: pour citer Aristote, elle est une energeia qui le rend en quelque sorte mortvivant. Son cadavre est animé, au sens étymologique: doté d'un «souffle vital» et d'une «âme» qui perdure dans la postérité. Nous retrouvons cette valeur universelle dans la civilisation et les récits grecs. David Le Breton rappelle que «mourir n'est rien pour un héros grec; l'horreur ne touche que celui qui a failli au combat par lâcheté»(Le Breton, 2006, p. 82). La gloire est d'ordre métaphysique et ne se réduit pas à un pur prestige social. Elle est une forme de dépassement de la mort, une manière d'entrer dans l'éternité de la mémoire. "Ce que le héros perd en honneurs, rendus à sa personne vivante, quand il renonce à la longue vie pour choisir la prompte mort, il le regagne au centuple dans la gloire dont est auréolé, pour tous les temps à venir, son personnage défunt », résume Jean-Pierre Vernant (1982, p. 53). Et chez les Indiens, de manière étonnante, nous retrouvons ces valeurs communes à la société occidentale médiévale: «Ce prophete parle à eux en public, les exhortant à la vertu et à leur devoir; mais toute leur science ethique ne contient que ces deux articles, de la resolution à la guerre et affection à leurs femmes », explique Montaigne (1580, I, XXXI). D'autre part, il est intéressant de constater que le terme de «cannibale» vient de caraïb, qui signifie «hardi » dans la langue locale des îles. À la fois européen et américain, c'est un mot métis lié à Christophe Colomb, qui semble avoir été impressionné par les récits de peuples victimes du cannibalisme de leurs voisins.

Montaigne fuit la mollesse de l'entredeux et de la grisaille. Aussi est-il admiratif de la hardiesse des guerriers indiens et de la radicalité de leurs combats sanglants où l'audace l'emporte sur la peur: "C'est chose esmerveillable que de la fermeté de leurs combats, qui ne finissent jamais que par meurtre et effusion de sang; car, de routes et d'effroy, ils ne sçavent que c'est» (I, XXXI). La crainte extrême des Indiens, observe Montaigne, n'est pas de mourir mais d'être mortifiés. Elle s'illustre par un fort sens de l'honneur: "la vaillance, c'est la fermeté, non pas des jambes et des bras, mais du courage et de l'ame» (Montaigne, 1580, I, XXXI).

Le cadavre est ambigu: d'une part, il est l'image inoubliable qui doit aider à apaiser le feu des vaines prétentions humaines; d'autre part, la conscience de son imminence ne doit pas entraver la vitalité ni la bravoure, car l'angoisse instinctive et pétrifiante de la mort est le poison des vivants. Dans certaines iconographies de la Renaissance, un squelette peut s'amuser et faire de malicieux clins d'œil, suggérant presque à son spectateur que le mort peut être animé. Les planches anatomiques du médecin André Vésale qui proposent une représentation humanisée des cadavres en sont une parfaite illustration. Elles appuient l'idée que pour pénétrer l'essence de l'humain il faut décharner le cadavre jusqu'aux os. Cette esthétique du dépouillement est voisine de la fascination de la nudité du sauvage, dont la reconnaissance sociale par sa communauté est fondée sur les seules valeurs de vaillance et de défi à la mort, et ne se manifeste pas par des atours. D'ailleurs, Montaigne clôt son chapitre «Des Cannibales» sur cette boutade: "Mais quoy, ils ne portent point de haut de chausses. » Le dénuement total des Indiens n'est pas sans rappeler la nudité d'Adam et Ėve, évoquée par la Genèse dans le cadre idyllique du paradis perdu des âges premiers:

C'est une nation [...] en laquelle il n'y a aucune espece de trafique; nulle cognoissance de lettres; nulle science de nombres; nul nom de magistrat, ny de superiorité politique; nul usage de service, de richesse ou de pauvreté; nuls contrats; nulles successions; nuls partages; nulles occupations qu'oysives; nul respect de parenté que commun; nuls vestemens; nulle agriculture; nul metal; nul usage de vin ou de bled (Montaigne, 1580, I, XXXI).

Dans cette énumération, Montaigne semble emporté dans un euphorique déshabillage de sa propre nation qu'il dépouille de tous ses oripeaux, au rythme tonique des anaphores qui s'enchaînent les unes après les autres, jusqu'à ce que le «nul» rejoigne le «nu»d'une société et peut-être même son ossature et sa légèreté première.

À l'inverse, Montaigne s'attriste de constater que «nous avons tant rechargé la beauté et richesse de ses ouvrages [ceux de mère nature] par nos inventions, que nous l'avons du tout estouffée» (Montaigne, 1580, I, XXXI). Il partage la pensée platonicienne qui subordonne l'art à la nature : «Toutes choses, dict Platon, sont produites par la nature, ou par la fortune, ou par l'art; les plus grandes et plus belles, par l'une ou l'autre des deux premieres; les moindres et imparfaictes, par la derniere » (I, XXXI). Montaigne n'hésite pas à s'attaquer à la rhétorique qui recouvre le langage d'un artifice trompeur: "car les fines gens [...] ne vous représentent jamais les choses pures; ils les inclinent et masquent selon le visage qu'ils leur ont veu»(I, XXXI). Au contraire, il voudrait «que chacun escrivit ce qu'il sçait», qu'il y ait «narration particuliere» et "particuliere science», indépendamment de la "voix commune», afin de donner le primat à l'« experience». 
Son idéal d'écriture, largement emprunté aux Anciens, rejoint le modèle des Indiens à qui il prête un «doux langage » et pur au "son aggreable, retirant aux terminaisons Grecques» (I, XXXI).

En des temps où l'Église surveillait de très près l'utilisation de cadavres pour la dissection et enseignait le caractère sacré du corps humain, Montaigne propose une approche téléologique du cadavre. Ce dernier s'autodétermine dans une continuité temporelle et n'est plus uniquement assujetti à sa destinée théologique première, happé par l'intemporel des Cieux ou des Enfers. Il reste ancré dans la matérialité terrestre en dépit de son inéluctable précarité: mangé, le cadavre conserve une forme d'immortalité, participant au cycle du renouvellement naturel; anatomisé, il entre dans le champ du savoir et assouvit un profond désir de connaissance du vivant.

Enfin, le livre ne peut-il pas être luimême considéré comme le cadavre de son auteur, comme les restes qu'il nous lègue? Ne dit-on pas dans le langage courant: "J'ai dévoré Montaigne»? Antonomase signifiante pour notre sujet et qui corrobore parfaitement les propos de Montaigne lui-même: "Je suis moy-mesmes la matiere de mon livre ${ }^{6}$, ou encore : "Me trouvant entierement despourveu et vuide de toute autre matiere, je me suis presenté moy-mesmes à moy, pour argument et pour subject » (Montaigne, 1580, II, VIII). Montaigne ne se livre-t-il pas ainsi au cannibale lecteur? Il songe même à s'offrir dénudé et prêt à consommer: "J'aimeray quelqu'un qui me sçache deplumer» (Montaigne, 1580, II, $\mathrm{X})$, confie-t-il; ou encore: "Je t'asseure que je m'y fusse tres-volontiers peint tout entier, et tout nud ${ }^{7}$. Montaigne prône d'ailleurs une écriture nue et organique, dont l'inspiration n'émanerait pas d'un souffle divin comme dans la tradition platonicienne, mais de son propre corps, de ses propres sens, de son propre souffle. Il défend une écriture immanente à la nature et non transcendante: "Je m'estudie plus qu'autre subject. C'est ma metaphisique, c'est ma phisique» (Montaigne, 1580, I, «Au lecteur»). Dans le chapitre «De l'exercitation », l'approximation de l'essayiste lui permet de se connaître et d'approcher l'inconnaissable: la mort. La mise en écrit de ses tâtonnements lui permet d'imposer une forme à l'informe, à l'ineffable de la mort. Son essai est alors une tentative de figer ses pensées face à la fluidité de l'expérience réelle, soit le passage dans la mort impossible à raconter. Le tâtonnement rhétorique se meut alors en cadavre textuel et Montaigne ne peut présenter à son lecteur qu'une simulation de la mort, ou plutôt son avatar, c'est-à-dire un simili-cadavre : «Je m'estalle entier: c'est un Skeletos où, d'une veue, les veines, les muscles, les tendons paroissent, chaque piece en son siege» (Montaigne, 1580, II, VI). Selon Lawrence D. Kritzman, Montaigne réalise une résurrection qui n'en est pas une: "cette prothèse rhétorique projette la simulation d'une fin éprouvée et la mise en place d'une immortalité paradoxale dans le tombeau scriptural où gît la matière morte de son écriture ${ }^{8}$.

En se nourrissant du livre-cadavre de Montaigne, le lecteur a souvent la surprise de tomber sur des morceaux d'autres auteurs que sont les nombreuses citations qui parcourent son œuvre. En effet, Montaigne est lui-même nourri d'auteurs classiques qu'il incorpore après sélection: Plutarque, Sénèque, Lucrèce, Catulle, Horace, Lucain et surtout Virgile... Il en absorbe le «suc» et la «substance» et «leur instruction est la crème de la philosophie». Montaigne a ses goûts, il n'apprécie guère l'écriture de Cicéron pleine de «longueries d'apprêts». En somme, comme l'écrit Agnès Minazzoli, il «mange ses pères d'écriture tout crus » (Minazzoli, 1991, p. 225). Cette rhétorique de la citation est le véritable noyau du texte. L'innutrition sera la méthode: digérant le grec, les Romains ont fait de leur langue l'égale du grec. À la Renaissance, il faut en faire autant avec les langues nationales. L'imitation est un art de la greffe qui constitue un véritable rituel dans la culture humaniste, une sorte de structure stable, de point d'ancrage dans un monde perçu par ailleurs comme en pleine révolution et en grande instabilité. La réécriture se manifeste donc d'emblée comme une structure conversationnelle. Ce processus de digestion et d'assimilation généreuse de la culture de l'Autre s'oppose à la culture du vomissement. Claude Lévi-Strauss distingue en effet les sociétés anthropophages des sociétés «anthropèmes» (du grec émein, «vomir»), qui expulsent l'Autre du corps social au lieu de l'absorber afin de le neutraliser et d'en tirer profit. À la Renaissance, la lecture ouvre au monde, aux autres, aux hommes considérés à tort comme étrangers : il s'agit de comprendre ce qui est nouveau jusque dans la découverte des textes anciens.

Les auteurs ne sont-ils pas les maillons d'une vaste chaîne alimentaire? Et la lecture n'est-elle pas un processus de mastication incessante? En somme, la littérature est un grand banquet dont les livres sont les mets, où les lecteurs sont les convives, et où trônent certaines figures auctoriales et totémiques. Hérodote décrit ainsi le rituel des Issédones lors d'une disparition paternelle:
Voici, dit-on, les coutumes des

Issédones. Lorsqu'un homme a perdu son père, tous ses proches lui amènent du bétail; les animaux sont sacrifiés et dépecés, puis on découpe également le cadavre du père, on mêle toutes les viandes et on sert un banquet. $\mathrm{La}$ tête du mort, soigneusement rasée, vidée, est recouverte de feuilles d'or et devient pour eux une image sacrée à laquelle on offre tous les ans des sacrifices somptueux. Le fils rend cet honneur à son père (Hérodote, 2008, IV, XXVI).

L'écriture est une communication en différé, voire une communication avec les morts. Comme l'image et comme les emplois du cadavre que nous avons largement disséqués, l'écriture révèle une présence-absence. Incontestablement, elle ne cesse de ressusciter nos morts. Montaigne sait le péril qui menace ces nations découvertes au Brésil et est conscient d'apporter une trace de ce qui a été, à la manière d'un anthropologue; il restitue un témoignage, attesté d'ailleurs par d'autres auteurs ${ }^{9}$. Même si c'est bien notre société européenne qu'il étudie et qui est sa principale focale, les Tupinambas n'étant que le contrepoint didactique qui rend davantage visibles nos propres failles. Il manifeste donc bien une volonté d'éducation de ses contemporains, en leur tendant un miroir qui laisse entrevoir une forme d'idéal social.

Enfin, l'écriture peut avoir valeur testamentaire: offrir son texte - et à travers lui, son corps - à des générations de lecteurs, c'est pour l'auteur une manière d'apprivoiser la mort. Sans cette relation à l'Autre, même par-delà la mort, sans cette dynamique d'échanges, le livre n'est qu'un tombeau. Il y aurait chez Montaigne la tentation de faire coïncider ainsi le mot et la chose: que le mot ait une texture quasi organique. Le cadavre étant alors le lieu même de la langue; un lieu réflexif d'interrogation métapoétique. Si le mot tue la chose, alors le cadavre par sa matérialité lui redonne un souffle nouveau. Il est ambivalent comme le langage: ses deux facettes de part et d'autre de la frontière du réel sont le symétrique du couple linguistique signifiant / signifié. Représentant le mot et la chose, le cadavre est un corps, une présence, mais aussi un corps non investi, une absence. Comme le résume Antoine Compagnon, «Montaigne pose ainsi une équation stricte entre le livre et l'auteur, affirme leur identité et leur consubstantialité. Par une innovation radicale, la matière et la manière du livre équivalent à la vie de l'homme» (Compagnon, s.d.). 
L'immortalité terrestre du cadavre, autopsiée dans cette enquête, est à bien y réfléchir purement langagière : c'est parce que les Tupinambas attestent avoir mangé des ancêtres de la tribu voisine que ceux-ci demeurent toujours en eux. Le cadavre par son creux, par son dénuement, nous donne à voir ce qui nous habite - notre essence. Il est un corps vide qu'il faut gloser afin de le fixer et d'éviter son évanouissement. L'épitaphe lui est consubstantielle et le maintient dans la légende. Se développe très fortement à la Renaissance une littérature de l'épitaphe, avec la vogue des tombeaux poétiques; notons aussi que le terme "légendaire» est emprunté au latin médiéval legenda, qui désigne les «choses à lire» et en particulier les «vies de saints ». Selon Maurice Blanchot, il y a un rapport très étroit entre la mort et l'image: la mort, c'est l'image. Pour lui, le cadavre est une image «qui ne ressemble à rien » (Blanchot, 1948, p. 173) ; son épitaphe lui sert de légende et lui permet de recouvrer une ressemblance, un référent, et de demeurer dans une histoire et donc une mémoire. C'est précisément cette parole qui le rend toujours vivant parmi les mortels. Si le cadavre doit au vivant son immortalité, il est en échange donneur de «leçon», mot que l'étymologie fait également dériver de legere.

Enfin, le langage crée le monde en le nommant, mais détient aussi le pouvoir de l'anéantir, par cette désignation même. Comme le langage, le cadavre à la Renaissance fait signe: contrepoint à la vie, il la signale. Le cadavre est pour les humanistes une source de savoir et de connaissance; mieux encore, il enseigne, il est une leçon de vie. Comme le cadavre a besoin de nous, nous ne pouvons vivre sans nos cadavres, car par leur matérialité même ils nous empêchent de fuir la réalité de la mort. Or, sentir la mort, c'est être vraiment dans la vie et non dans son illusion.

\section{Bibliographie}

BLANCHOT, M. (1948). L'Arrêt de mort, Paris, Gallimard, coll. "Imaginaire».

COMPAGNON, A. (1993). Chat en poche, Montaigne et l'allégorie, Paris, Seuil.

COMPAGNON, A. (s.d.). "Qu'est-ce qu'un auteur? Sixième leçon: Les jeux de la Renaissance», en ligne, <http://www. fabula.org/compagnon/auteur6.php $>$, consulté le 2011-01-15.

DANTE, A. (1985). L'Enfer, trad. française par Jacqueline RISSET, Paris, Flammarion.

DEBRAY, R. (1992). Vie et mort de l'image, Paris, Gallimard, coll. «Folio Essais».

DESAN, P. (2008). Montaigne: les formes du monde et de l'esprit, Paris, Presses universitaires Paris-Sorbonne.
DE SOUZA FILHO, J.A. (2005). Civilisation et barbarie en France au temps de Montaigne, Lille, Atelier national de reproduction des thèses.

HÉRODOTE (2008 [1985]). L'enquête, trad. par Andrée BARGUET, Paris, Gallimard, coll. «Folio classique».

KRITZMAN, L.D. (2009). The Fabulous Imagination: On Montaigne's Essays, Columbia University Press.

LE BRETON, D. (2006). "Le cadavre ambigu: approche anthropologique ", dans L'Esprit du Temps, $\mathrm{n}^{\circ}$ 129, "Études sur la mort», p. 79-90.

LÉRY, J. DE (1992 [1578]). «Comment les Américains traitent leurs prisonniers pris en guerre, et les cérémonies qu'ils observent, tant à les tuer qu'à les manger", dans Histoire d'un voyage fait en la terre du Brésil, chap. XV, éd. présentée par Franck LESTRINGANT, Presses du Languedoc, Max Chaleil.

MINAZZOLI, A. (1991). "Manger de l'homme? Le cannibalisme de Montaigne et après... », dans M. GAGNEBIN et J. MILLY (dir.), L'affrontement et ses images, Seyssel, Champ Vallon, p. 212-230.

MONTAIGNE, M.E. DE (1965 [1580]). Les Essais, éd. établie par Pierre VILLEY et Verdun Louis SAULNIER, Paris, Presses universitaires de France.

NAKAM, G. (1991). Montaigne. La manière et la matière, Paris, H. Champion.

RABELAIS, F. (1994). Le Tiers Livre, dans Euvres Complètes, éd. présentée et annotée par Mireille HUCHON, Paris, Gallimard, coll. «Bibliothèque de la Pléiade».

VERNANT, J.-P. (1982). «La belle mort et le cadavre outragé », dans La mort, les morts dans les sociétés anciennes, Paris, Maison des Sciences de l'Homme et Cambridge University Press

VÉSALE, A. (1543). De humani corporis fabrica, Bâle, Johannes Oporinus.

\section{Notes}

1. Michel Eyquem de Montaigne (1580), Essais, Livre I, chap. XXXI, «Des Cannibales». Nous utilisons l'édition établie par Pierre Villey et Verdun Louis Saulnier, Paris, Presses universitaires de France, 1965.

2. Pensons ici au célèbre personnage d'Ugolin, chez Dante, qui en est réduit à manger ses enfants : " Père, nous souffririons bien moins / si tu nous mangeais; tu nous as vêtus / de ces pauvres chairs; enlève-les nous. " / Je me calmai alors pour ne pas aggraver / leur peine; pendant deux jours nous fûmes tous sans voix; / ah terre cruelle, que ne t'ouvristu? » (Ugolin voit mourir ses enfants, un à un, pendant deux jours.) «Et puis ce que la douleur ne put, la faim le put. / Quand il eut dit ces mots, le regard tors, / il reprit le malheureux crâne avec ses dents / qui mordirent l'os comme celles d'un chien » (Dante, 1985, L'Enfer, XXXIII, 62-78, p. 299-301).

3. À la Renaissance, certains soutiennent la thèse d'une sensibilité ininterrompue du cadavre: forme différente de vie qui continue- rait à se manifester au-delà de la mort et qui perdurerait dans la chair. D'autres évoquent la présence de l'âme, faisant de l'homme un composé dont la mort ne disloque pas les parties. Plus tard, un médecin luthérien allemand, Garmann (1640-1708), défendra cette idée dans un traité de médecine paru après sa mort. Il avance comme preuve de la sensibilité du cadavre la tradition judiciaire de la cruentatio, c'est-à-dire de la faculté que conserve l'homme victime d'un crime de se mettre à saigner lorsqu'il est mis en présence de celui qui a attenté à sa vie. Garmann évoque aussi les mouvements singuliers du cadavre, les bruits qui s'en échappent, les ongles ou les poils qui continuent à pousser.

4. À ce propos, notons que le témoignage de Montaigne concorde avec celui de Jean de Léry: «Ils les gardent plus ou moins de temps, tant y a néanmoins qu'après les avoir engraissés comme pourceaux en l'auge, ils sont finalement assommés et mangés » (Léry, 1992 [1578]).

5. À propos des Scythes, Hérodote (mentionné par Montaigne) décrit un rite semblable sur le traitement de la peau des cadavres: «Beaucoup aussi prélèvent sur les cadavres de leurs adversaires la peau de la main droite avec les ongles pour en faire des couvercles de carquois; la peau humaine est assurément épaisse et lustrée, supérieure peut-être à toutes les autres en blancheur et en éclat. Beaucoup écorchent même des hommes tout entiers et tendent les peaux sur des cadres de bois qu'ils juchent sur leurs chevaux pour les exhiber à la ronde» (Hérodote, 2008, Livre IV, XXVI).

6. Montaigne, Essais, Livre II, chap. VIII, «De l'affection des pères aux enfans".

7. Montaigne, Essais, Livre I, «Au lecteur».

8. Lawrence D. Kritzman, «La mort chez Montaigne », conférence à l'EHESS, mai 2010.

9. Montaigne évoque notamment Jean de Léry, Antoine Thevet et Fransisco Lopez de Gomara. 